\title{
Immersive Virtual Reality for Earth Sciences
}

\author{
Ilario Gabriele Gerloni, Vincenza Carchiolo \\ Department of Electrical, Electronic and Computer Engineering, University of Catania, Italy \\ Email: ilario.gerloni@gmail.com
}

Fabio Roberto Vitello, Eva Sciacca, Ugo Becciani, Alessandro Costa, Simone Riggi

Astrophysical Observatory of Catania, Italian National Institute for Astrophysics (INAF), Italy

Email: fabio.vitello@inaf.it

\author{
Fabio Luca Bonali, Elena Russo, Luca Fallati, Fabio Marchese, Alessandro Tibaldi \\ Department of Earth and Environmental Sciences, University of Milan Bicocca \\ Email: alessandro.tibaldi@unimib.it
}

\begin{abstract}
This paper presents a novel immersive Virtual Reality platform, named ARGO3D, tailored for improving research and teaching activities in Earth Sciences. The platform facilitates the exploration of geological environments and the assessment of geo-hazards, allowing reaching key sites of interest (some of them impossible to be reached in person) and thus to take measurements and collect data as it can be done in the real field. The target audience of ARGO3D encompasses students, teachers and early career scientists, as well as civil planning organisations and non-academics. The overall workflow for real ambient reconstruction, processing and rendering of the virtual ambient is presented, as well as a detailed description of the VR software tools and hardware devices employed.
\end{abstract}

\section{INTRODUCTION}

D UE TO technological advances in instruments and detectors over the last two decades, there has been a true explosion in both the amount and the complexity of scientific data, an exponential trend that will continue at ever increasing rate in the coming years. The concept of Big Data - defined not only as datasets too large to be processed with today's tools and methods but also (and perhaps more importantly) as datasets too complex to be effectively dealt with - permeates our world. Over the last decade, hundreds of thousands measurements involving complex 3D datasets of geological structures of the active seismic zones have been collected. The development of new instruments results in increasingly high resolution images and datasets, e.g. from Unmanned Aerial Vehicle (UAV) surveys. These data offer unique opportunities to build long term capacity for geo-hazard maps and studies never realised so far.

Virtual reality (VR) allows to generate virtual environments which gives the feeling of being elsewhere, within a real place. It can be considered as an advanced form of humancomputer interface that allows to interact with a realistic virtual environment generated using interactive software and hardware.

The "Virtual Reality", starting from the 60's, has evolved in different manners becoming more and more similar to the real

This work is supported by MIUR Accordo di programma ACPR15T4_00098 world. Nowadays, two different kinds of VR can be identified: non-immersive and immersive. The former is a desktop-based environment that can simulate places in the real or imagined worlds [11]; the latter takes the idea even further by giving the perception of being physically present in the non-physical world. The non-immersive VR can be based on a standard computer (using e.g. mouse, keyboard or joysticks), whereas the immersive VR is still evolving as the needed devices are becoming more user friendly and economically accessible.

In the past, there was a major difficulty about using VR equipment such as a helmet with goggles, while now new devices are being developed to make usability better for the user. VR is based on three basic principles: Immersion, Interaction, and User involvement with the environment. It offers a very high potential in education [5], [1], industry [13], research [3] and scientific collaboration [10], [8] by making the learning experience more motivating and engaging, and facilitating the investigation of complex scenarios. Up to now, the use of immersive VR in research, educational games and scientific dissemination has been limited due to the high price of the devices and their non user-friendly usability. At the moment, new virtual reality headsets like the commercial Oculus Rift ${ }^{T M}$ or smartphone headsets (e.g. Samsung Gear VR), make it possible to access immersive VR in lots of research, educational and dissemination contexts.

This paper presents the first platform for immersive VR in Earth Sciences, here named ARGO3D (Augmented and virtual reality for geology and geophysics - http://argo3d.unimib.it/). Our immersive VR platform facilitates the survey of geological environments and related geo-hazards assessment; the target audience regards early career scientists, civil planning organisations, as well as academic (e.g. students and teachers) and non academic people. Virtual 3D visualization and navigation allows players to fully explore areas of interest for geo-hazards (e.g. the crater of an active volcano), and, thanks to the data collected (and observations) by virtual exploration, to better define and interpret the geological phenomena affecting the selected site. Thus, players will be able to reach more holistic and comprehensive concepts, as the main specific features 
will be viewed from many different scales and perspectives. Moreover, students exhibit conceptual difficulty in interpreting various $2 \mathrm{D}$ diagrams and photos, showing $3 \mathrm{D}$ phenomena and geological site. Incorporating 3D visualizations into teaching earth related sciences can help the students in learning activities thanks to a wider spatial perspective and thinking, and may help them in improving their spatial ability.

\section{SCIENTIFIC BACKGROUND}

The use of virtual and augmented reality is a novel and extremely innovative method of 3D immersive approach for research and teaching activities in Earth Sciences. Marine and terrestrial environments will be brought directly into laboratories and classrooms giving the researchers and students the feeling of playing a videogame, instead they are exploring real geological key sites. Geological environments are rendered by means of the Structure from Motion (SfM) Photogrammetry technique. 3D scenarios are derived from UAVs regarding terrestrial environments and from submersibles (ROV) for marine environments, with exceptionally high-detailed images.

The ARGO3D platform is tailored with three main purposes: overcoming problems in research, as well as increasing the quality of training and outreaching activities in Earth and Environmental Sciences.

Regarding the research activity, the best way to study geo-hazards in terrestrial and submarine environments (such as landslides, volcanic eruptions, erosion, floods and recent surface seismic ruptures) is to examine rock outcrops and key sites directly in the field. Due to logistic conditions, this activity is often complicated or even impossible: for example, it is not achievable to directly explore the crater of an active volcano due to toxic gases and high temperatures whereas some peculiar geological spots are too far to be reached on foot or by car. Furthermore, very high vertical outcrops are difficult to be analysed in all their spatial extension.

Virtual reality represents a new way to bypass these problems, giving to the researcher the possibility to easily reach key sites of interest and thus to take measurements and collect data, as it can be done in the field. ARGO3D will allow both students and teachers to analyse environments and processes that would be impossible to observe in person.

In regard to the training and outreach activity, teachers will lead the students into virtually-reconstructed 'hard to reach' environments, explaining them the best ways to enjoy the activity and the geological meaning of the different features that appear on the screen. The development of useful teaching and training tools will allow each student to explore in real time virtually-reconstructed $3 \mathrm{D}$ environments, to take pictures of significant geological features, to collect basic geological data, as well as to virtually fly above the area and to export features to a GIS environment for further analysis. At the same time, the other students/people will have a chance to observe, just like in a $3 \mathrm{D}$ cinema/movie, how their colleagues navigate and interact with the environment. These tools will improve the students observation and field mapping skills, and their

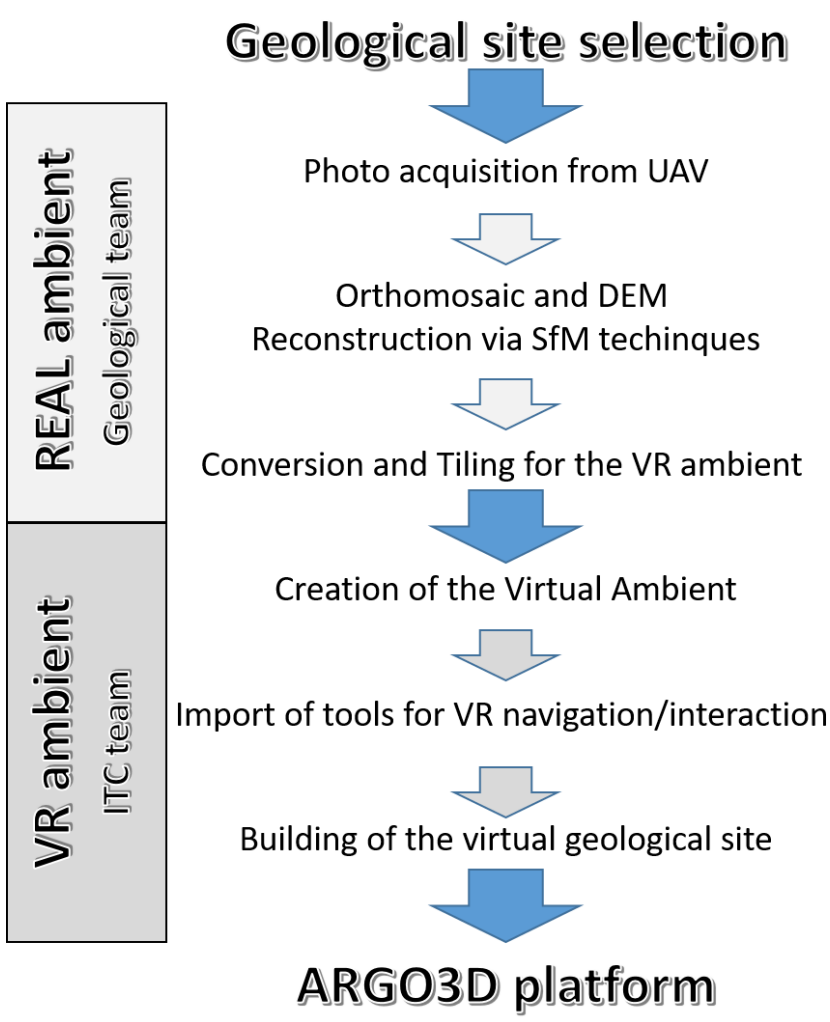

Fig. 1. General workflow describing the main steps involved for performing geological site reconstruction, processing and rendering from the real ambient into the VR ambient.

knowledge and understanding of areas subjected to natural hazards.

\section{Data Collection AND PRocessing}

The overall workflow describing the main steps involved for performing geological site reconstruction, processing and rendering from the real ambient into the VR ambient is shown in Figure 1. Two teams are currently working closely to virtualize the geological sites of interests: geologists and ITC specialists. Geologists are in charge of performing the first three steps that are described in this section.

The input files for the creation of 3D reconstructed environments (in terms of terrain and texture) have been generated thanks to the Structure from Motion algorithms (SfM) applied to Unmanned Aerial Vehicle (UAV or drone)-captured images (e.g. [4]). Such approach involves three main processes (see the first three steps of Figure 1): i) acquisition of a set of images collected in order to cover the selected geological site (with an overlap greater than $60 \%$ ); ii) processing of these images through a SfM software that leads to the creation of a orthomosaic and a digital elevation model (DEM) as final products; iii) conversion and tiling of the orthomosaic and DEM in a format compatible with Unity.

For the image acquisition we used a quadcopter, DJI Phantom 4 (Fig. 2A), one of the most used and versatile UAVs. They have been collected along a defined flight path $(50 \mathrm{~m}$ of 
altitude) in order to cover the whole selected geological key site with an overlap of $90 \%$ (Fig. 2B). In order to accurately georeference the resulting model, in addition to the GPS photo location from the drone, real-world coordinates of, at least, four Ground Control Points (GCPs) have been established within the surveyed area (e.g. [7]; [14]; [15]).

Regarding the SfM processing, UAV-captured photos have ${ }^{2}$ been processed with the use of Agisoft PhotoScan ${ }^{1}$, a $\mathrm{SfM}^{3}$ software. The SfM technique allowed to identify matching features in different images, collected along a defined fly path, and combine them to create a sparse and dense cloud (Fig. 2B), a mesh, an orthomosaic and a DEM (Fig. 2C-D) (further details in [12] and [15]). Our model is made of $20722 \times 20722$ pixels, with a corresponding pixel size of $0.02 \mathrm{~m}(20 \mathrm{~mm})$, the areal extension in an E-W and N-S direction is equal to 414.44 $\mathrm{m}$. Both the orthomosaic and DEM are in GeoTiff file format.

GeoTIFF file format is widely used for raster imagery and aerial photography since it contains georeferencing information, necessary to establish the exact spatial reference of the file, such as the datum and UTM zone; in our case, we used WGS84 datum and the UTM zone is $28 \mathrm{~N}$. The DEM results as a GeoTiff with each pixel associated with altitude value of the real ambient (Fig. 2D), whereas the resulting orthomosaic is composed by pixels each representing the RGB color of the real ambient (Fig. 2C). Both of them have been exported with the same areal extension and pixel size $(20 \mathrm{~mm} /$ pixel $)$; NULL values in the DEM and orthomosaic have been filled by interpolation (Fig. 2D) and RGB pixel colour from satellite images $(50 \mathrm{~cm} /$ pixel - image from Worldview-2 sensor, Catalogue ID 1030050051F92D00) (Fig. 2C), respectively.

In order to work as input file for Unity, the DEM must be converted in gray scale 16 bit RAW file (which is a format compatible with most image and landscape editors) with minimum value corresponding to 0 and maximum value to 65536 , corresponding to a range of $14.029 \mathrm{~m}$ in the real world. Due to the high resolution, in terms of pixel size, of our model, we chose to implement this conversion considering altitude and areal extension of the model in $\mathrm{mm}$. After that, we produced tiles of $512 \times 512$ pixels (required to support mobile devices) for the orthomosaic (Fig. 2E) and $513 \times 513$ pixels for the RAW file representing the DEM (Fig. 2F). The additional bit along $\mathrm{x}$ and $\mathrm{y}$ represents an overlap for a better merging of the tiles in Unity environment. In case of the selected key site, it results in $41 \times 41$ tiles, already flipped/rotated for the best import in Unity, each one with an extension of 10240x10240 $\mathrm{mm}$.

Each tile is finally associated with a descriptive text file, which adds information about the real environment depicted in the data previously mentioned. The essential information these files must contain are: longitude ("XWorldLimits" keyword contains leftmost one and rightmost one for the current tile), latitude ("YWorldLimits" keyword contains lowest one and highest one for the current tile) and elevation ("MinAlt" keyword is the minimum and "MaxAlt" keyword is the maximum

\footnotetext{
${ }^{1}$ Agisoft PhotoScan: http://www.agisoft.com/
}

for the current tile). These text files are formatted as JSON strings, used to calculate the position of the player in the real world coordinates.

An example of metadata (Fig. 2D) is provided below:

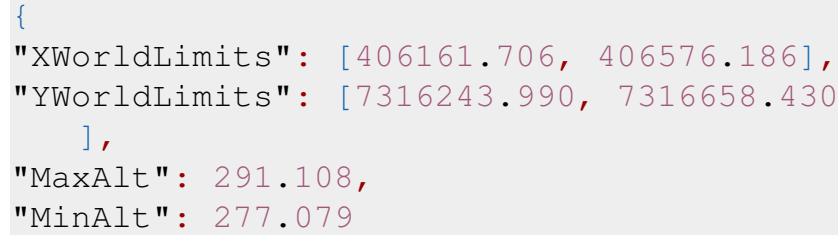

\section{ARGO3D PLATFORM}

\section{A. General Description}

In order to experience the software properly, the player must wear the VR headset and hold the input peripherals before running the application. At this stage, he also must sit in front of the motion sensor, so that all the startup settings, like default position of the user and peripherals in real world space, are completed.

When the virtual environment is loaded, the user can move and rotate his head to look at the computer-generated objects around himself. As said by Palmer Luckey in ${ }^{2}$, there are various infrared lights on the headset, that are used by the sensor to track the position and rotation of the user. When he moves his head, the software rotates the internal camera in order to simulate an immersive well-rounded vision of the environment.

To navigate in the scene, the player can use left hand "Thumbstick" placed on one of the provided input peripherals. These inputs are one for each hand, so multiple functionality are available for various scenarios, such as changing view and velocity through the scene (see subsection IV-B). These must be in the range of sensor placed in front of the peripherals, in order to be localized and rendered in the virtual scene.

\section{B. Use Cases}

The player has currently three possible modes to navigate in the scene, called "Walk Mode", "Flight Mode" and "Drone Mode" (Fig. 3). He can switch between these modes in every moment.

When the user is in "Walk Mode" (Fig. 3A) he behaves like a moving rigid body, so he's subject to gravity and can navigate the scene stick to the ground, or doing little jumps, thus experiencing the scene in realistic and immersive way. He can walk in a certain direction and look around thanks to the VR tools and, also, regulate his height with two special keys. This is the default mode, the one the user is in at the beginning.

In "Flight Mode" (Fig. 3B) the user can watch details that can't be observed or reached otherwise, and, at the same time, has a clear view from above of the entire scene. In

\footnotetext{
${ }^{2}$ https://www.vrfocus.com/2015/06/palmer-luckey-explains-oculus-riftsconstellation-tracking-and-fabric/
} 

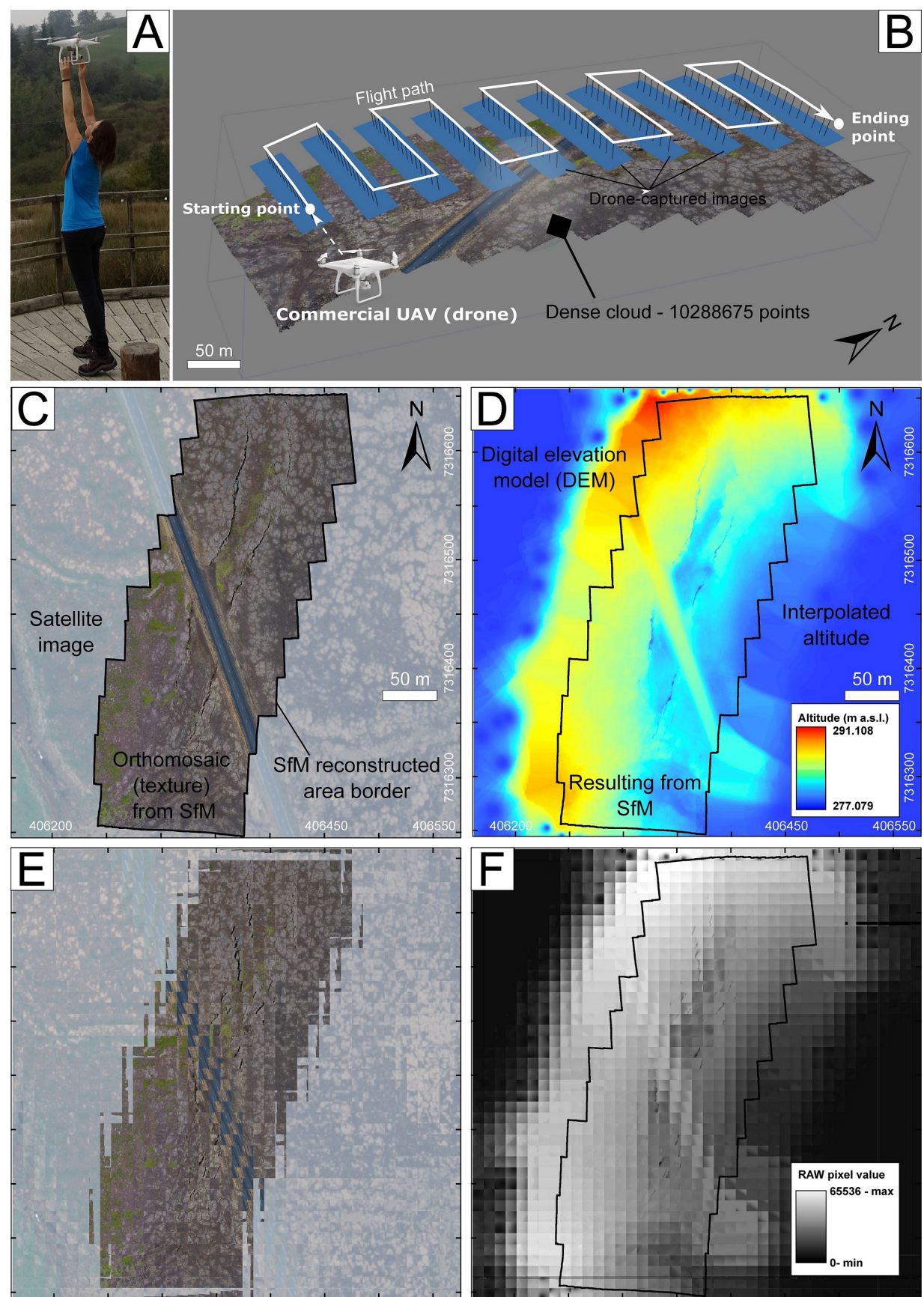

Fig. 2. Main steps involved in 3D reconstruction and processing for the input of the ARGO3D VR platform. (A) UAV (DJI Phantom 4) used for photo collection, person for scale. (B) 3D representation of the flight path, drone-captured photos (with location and orientation) and the dense cloud (resulting from SfM approach). (C) Orthomosaic resulting from the SfM processing (within the black border) characterized by the high resolution of $0.02 \mathrm{~m} /$ pixel. Outside the black border, pixels are from high-resolution satellite image $(50 \mathrm{~cm} /$ pixel - image from Worldview-2 sensor, Catalogue ID 1030050051F92D00). The whole area is scaled at the best pixel resolution $(0.02 \mathrm{~m} /$ pixel). (D) DEM resulting from SfM processing (within the black border) characterized by the high resolution of $0.02 \mathrm{~m} /$ pixel. Outside the black border, DEM values result from IDW interpolation. The whole DEM is scaled at the best pixel resolution (0.02 $\mathrm{m} / \mathrm{pixel}$ ). (E) Tiled texture and DEM (F) ready to be imported in Unity. The tiled texture is in JPG format, DEM values have been converted in Grayscale 16 bit RAW format. 

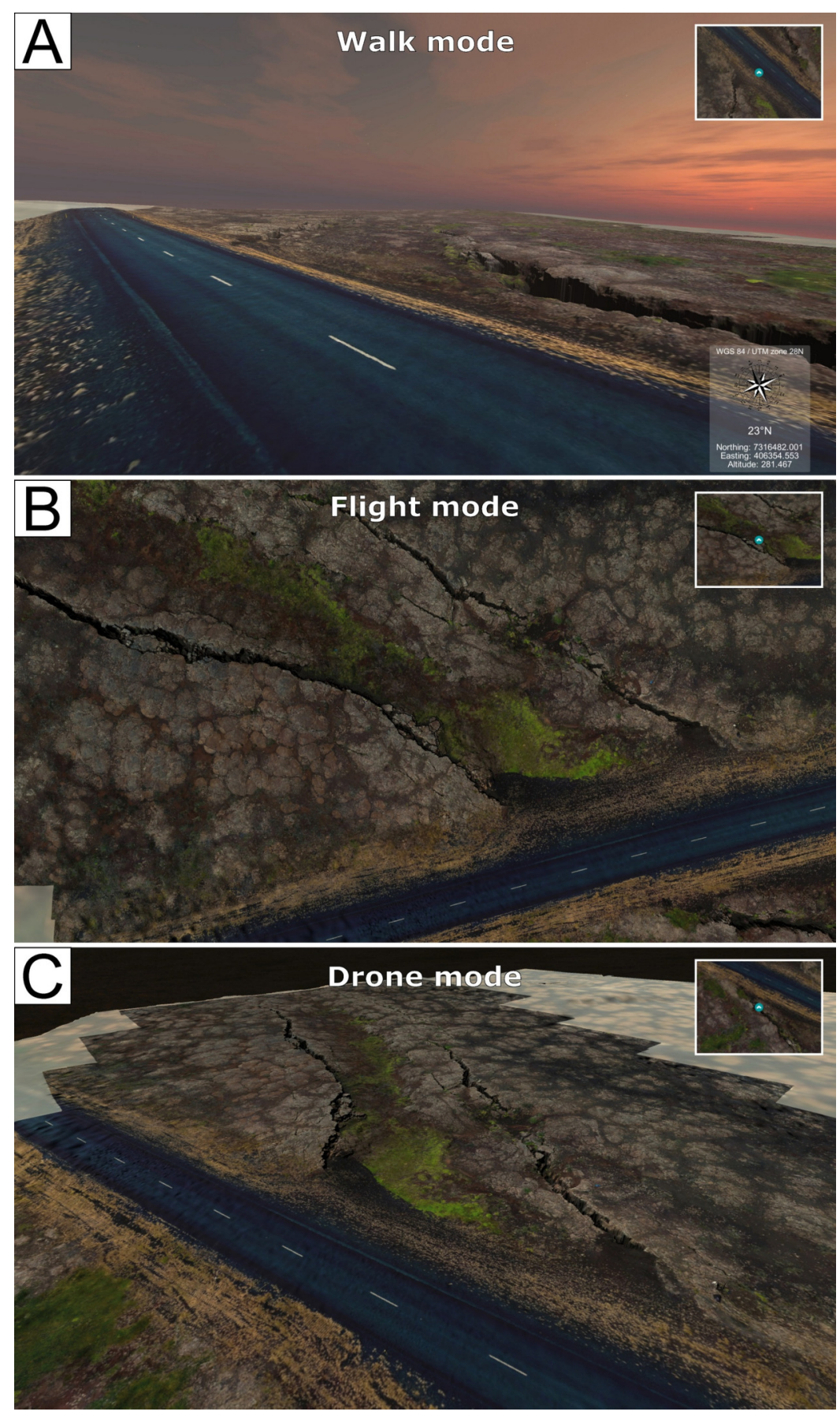

Fig. 3. Three modes of exploration are available in the immersive-VR ARGO3D platform: (A) Walk (B) Flight and (C) Drone mode. Inset in the upper right corner represents location of the player within the VR ambient (blue circle); in the lower right corner, GPS data are reported, they regard easting and northing (in UTM format), altitude (m), as well as the compass and the azimuth of where the player is looking. Road for scale. 
this mode, he relocates from the ground position to a certain ground clearance, and moves parallel to the ground as he's flying. The view is oriented orthogonally to the terrain, as the user can look at it from above, experiencing the whole comprehension of the scene. Two special keys allow to get closer or further from the ground, in a range of given values of ground clearance (this to avoid the user to be disoriented being too near to the ground or too far from it). When the user leaves "Flight" mode, it is relocated to the same position he currently was but near to the ground. The subsequent ground clearance depends on the mode the user is switching to: if on "Walk Mode", he finds himself standing on the ground; on the contrary, if on "Drone Mode" he's at the minimum height allowed by this mode.

In "Drone Mode" (Fig. 3C) the user has both the ability to move free in the scene and to not be subjected to gravity. The aim of this mode is to make the user feel like he's a radio-controlled drone, so he can fly on peaks and move to a certain ground clearance, but without the constraint of being forced to look in only one direction. If he tries to get too close to the ground he feels something like an elastic barrier that prevents him from crashing on the ground and relocate him at the correct height. In this mode the user is faster than in the others. A special feature allows him to aim at a point of the scene with tracked controller and rapidly move up to that point by pressing a trigger button. This was meant to be a quick way to reach certain locations far from the user.

In every mode it is visible to the user a GUI that shows useful information: a map in which it is possible to seek user position in the scene (view from above); below the map is located text with real world coordinates (northing and easting) and real world altitude, relative to his position in every moment; below this text there's a compass with azimuth of where the player is looking.

\section{Technologies}

1) Hardware technologies: The tool on which Virtual Reality is implemented is Oculus Rift (Fig. 4). The Oculus Rift headset uses an OLED panel for each eye, each having a pixel resolution of $1080 \times 1200$. The high refresh rate, global refresh and low persistence allow the user to experience none of the motion blurring or judder that is experienced on a regular monitor. It uses lenses with a wide field of view. The separation of the lenses is adjustable by a dial on the bottom of the device, in order to accommodate a wide range of interpupillary distances. The same pair of lenses are used for all users, however there are multiple facial interfaces so that the user's eyes can be positioned at a different distance. This also allows for users wearing glasses to use the Rift, as well as users with widely varying facial shapes.

The Rift has full 6 degrees of freedom rotational and positional tracking. This tracking is performed by Oculus's Constellation used to track the position of the user's head as well as other VR devices, consisting of external infrared tracking sensors that optically track specially designed VR devices.

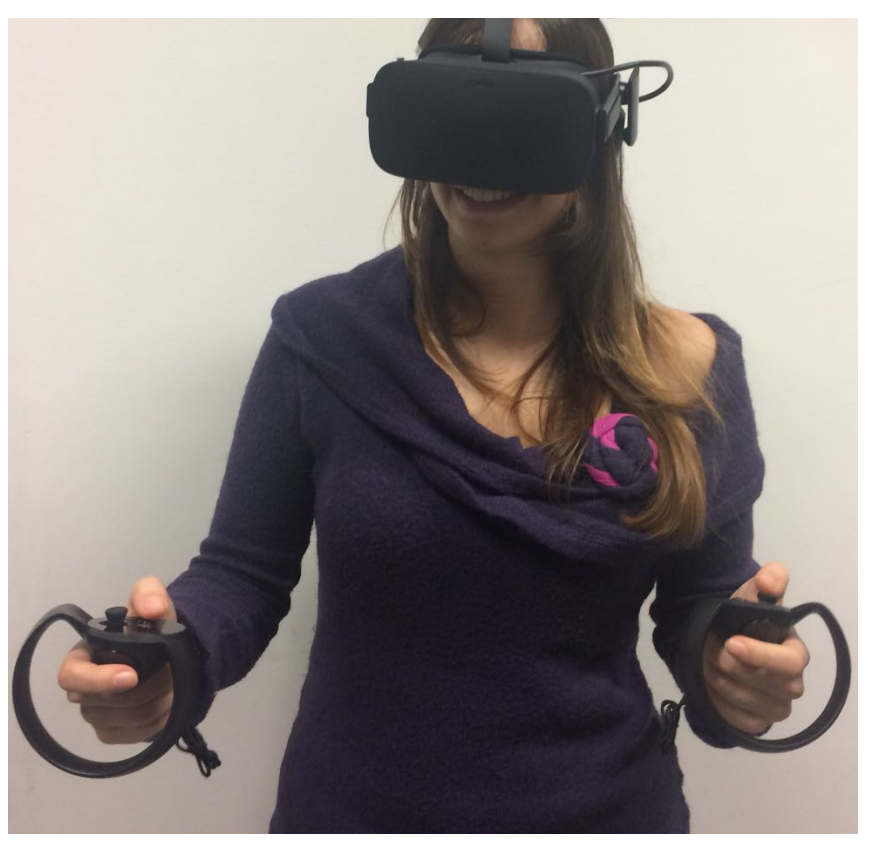

Fig. 4. Player using the Oculus Rift (VR headset) and the input peripherals.

The constellation sensor comes with a stand of a desk lamp form factor, but has standard screw holes and can be detached from this stand and mounted anywhere appropriate to the user. The Rift, or any other device being tracked by the system, is fitted with a series of precisely positioned infrared LEDs under or above the surface, set to blink in a specific pattern. By knowing the configuration of the LEDs on the objects and their pattern, the system can determine the precise position of the device with sub-millimeter accuracy and near-zero latency.

The Oculus Rift's motion controller system is known as Oculus Touch. It consists of a pair of handheld units, one for each hand, each containing an analog stick, three buttons, and two triggers. The controllers are fully tracked in 3D space by the Constellation system, so they may be represented in the virtual environment, and each controller features a system for detecting finger gestures the user may make while holding them.

2) Software tools: To develop all the scenes and to test the experience it's been chosen Unity3D v. 2017. Unity is composed of a set of windows to control various parts of the scene and development, such as the Scene Window, where it's possible to locate the assets as they'll be seen in the final version of the software, the Game Window, where it's possible to test the runtime behaviour of the software while editing it, and the Hierarchy, where the developer is able to keep track of all the active asset in the scene.

Every asset has a component that describes it. The essential one is the "Transform" component, which describes position, rotation and scale of the object in the scene. If an object is visible he must have a "Renderer" component, which can be a 2D or 3D Renderer, depending on purpose and design. Other important components are "Collider", which calculates where 
there's been a collision between objects and collects information about it, "Rigidbody", which simulates the behaviour of an object subject to kinematic and dynamic laws.

When a script, which implements an object-oriented paradigm, is created, it is attached to the object as a component. It's responsible of computation of data, but also of controlling and coordinating components, in order to obtain aimed behaviour in the scene.

\section{RELATED WORKS}

VR has been extensively used in geosciences. In this section we give an overview of some interesting recent use cases exploiting VR technologies.

Kinsland and Borst in [9] have presented the utility of $3 \mathrm{D}$ virtual reality systems in the interpretation of various 3D geologic/geophysical datasets. They have more than 10 years experience mainly exploiting large tiled display systems. In their studies they have identified some reasons that can lead to low utilization of the investigated systems for geological studies, i.e.: geologists would like to use their own desks to access other data useful for interpretation, they would require support from computer specialists, they experience motion sickness. Therefore, in the design of our ARGO3D platform we have tried to minimize these issues by improving the software usability and portability to common desktops with head mounted device and mobile devices.

Cerfontaine et al in [2] presented the workflow used to create immersive visualizations and spatial interaction for geophysical data with head mounted devices. They have used Unreal Engine 4 as VR tool for developing the platform to perform simple scene navigation (more interaction mechanisms are foreseen as future works). For the ARGO3D platform it was decided to use a different game engine and editor: Unity 3D 2017. This game engine was best-suited for our development, because the complexity of scripts and the weight of the computation is quite proportional to the amount of asset and functionality of the software. This is different from other game editors, which need more work and computational power to reach initial acceptable performances. Unity supports the object-oriented programming language $\mathrm{C \#}$, that is more modular, so it is possible to define different functionalities in different period of development, more simple and structured, so very easy to read and understand; C\# also has very good performance compared to his simplicity. Unity is much more documented on the web, so it's been easy to tackle most of the problems.

Finally, the authors in [6] discuss how VR technology could support geohazard research. In our project, we are further expanding the investigation of the application of VR for geohazards' assessment, by implementing the ARGO3D platform.

\section{CONCLUSIONS}

We presented the first results of our innovative research centered on experimenting and implementing a new approach designed to use the immersive VR for geo-hazards' assessment. Such research results in a platform, here named ARGO3D, that contains several ad-hoc tools, and will be soon available on http://argo3d.unimib.it/. Applications comprise both research and teaching activities in Earth Sciences, the target audience spans from academic to non-academic people. ARGO3D is designed to facilitate communicating geological environments and hazards to students, early career scientists, civil planning organizations and citizens.

We described the overall workflow for real ambient reconstruction, processing and rendering of the virtual ambient, the VR tools and hardware devices employed.

Our approach is aimed at giving the possibility of navigating into a 3D geological environment, where it is possible to observe in detail and measure objects of interest for Earth Sciences and geo-hazards in particular. This approach allows to re-create in laboratory real geological settings in order to share data and study key sites of interest at any time. Moreover, this allows to investigate also sites which are logistically difficult to reach in the field, or dangerous as, for example, active volcanic areas.

\section{ACKNOWLEDGMENT}

This work benefits from ESA Project Nr. 38829 (PI Fabio L. Bonali) and from fundings from the Italian Ministry of Education, Universities and Research: "Agreement University of Milan Bicocca - Consortium Cometa for the evaluation of leading-edge interactive technologies for improving teaching and popularization of science". This article is also an outcome of Project MIUR - Dipartimenti di Eccellenza 2018-2022.

\section{REFERENCES}

[1] Ajay Karthic B Gopinath Bharathi and Conrad S Tucker. Investigating the impact of interactive immersive virtual reality environments in enhancing task performance in online engineering design activities. In ASME 2015 International Design Engineering Technical Conferences and Computers and Information in Engineering Conference, pages V003T04A004-V003T04A004. American Society of Mechanical Engineers, 2015.

[2] Philippe A Cerfontaine, Anne-Sophie Mreyen, and Hans-Balder Havenith. Immersive visualization of geophysical data. In $3 D$ Imaging (IC3D), 2016 International Conference on, pages 1-6. IEEE, 2016.

[3] Ciro Donalek, S George Djorgovski, Alex Cioc, Anwell Wang, Jerry Zhang, Elizabeth Lawler, Stacy Yeh, Ashish Mahabal, Matthew Graham, Andrew Drake, et al. Immersive and collaborative data visualization using virtual reality platforms. In Big Data (Big Data), 2014 IEEE International Conference on, pages 609-614. IEEE, 2014.

[4] Bonali F.L., Tibaldi A., Marchese F., Fallati L., Russo E., Corselli C., and Savini A. Uav survey in volcano-tectonics: Methodology, best practice and application to the iceland rift. Journal of Structural Geology, Submitted.

[5] Laura Freina and Michela Ott. A literature review on immersive virtual reality in education: state of the art and perspectives. In The International Scientific Conference eLearning and Software for Education, volume 1, page 133. " Carol I" National Defence University, 2015.

[6] Hans-Balder Havenith, Philippe Cerfontaine, and Anne-Sophie Mreyen. How virtual reality can help visualise and assess geohazards. International Journal of Digital Earth, pages 1-17, 2017.

[7] MR James and Stuart Robson. Straightforward reconstruction of 3d surfaces and topography with a camera: Accuracy and geoscience application. Journal of Geophysical Research: Earth Surface, 117(F3), 2012.

[8] V Juř́ik, L Herman, P Kubíček, Z Stachoň, and Č Šašinka. Cognitive aspects of collaboration in $3 \mathrm{~d}$ virtual environments. The International Archives of Photogrammetry, Remote Sensing and Spatial Information Sciences, 41:663, 2016. 
[9] Gary L Kinsland and Christoph W Borst. Visualization and interpretation of geologic data in 3d virtual reality. Interpretation, 3(3):SX13-SX20, 2015.

[10] Danielle Oprean, Mark Simpson, and Alexander Klippel. Collaborating remotely: an evaluation of immersive capabilities on spatial experiences and team membership. International Journal of Digital Earth, 11(4):420-436, 2018.

[11] George G Robertson, Stuart K Card, and Jock D Mackinlay. Three views of virtual reality: nonimmersive virtual reality. Computer, 26(2):81, 1993.

[12] Cornelis Stal, Jean Bourgeois, Philippe De Maeyer, Guy De Mulder, Alain De Wulf, Rudi Goossens, Marijn Hendrickx, Timothy Nuttens, and Birger Stichelbaut. Test case on the quality analysis of structure from motion in airborne applications. In 32nd EARSeL Symposium: Advances in geosciences. European Association of Remote Sensing Laboratories
(EARSeL), 2012.

[13] JD Tibbett, FT Suorineni, and BK Hebblewhite. The use of virtual reality scientific visualisation for investigation and exploration of block cave mining system data. In Proceedings of the Virtual Reality and Spatial Information Applications in the Mining Industry Conference, $2015 \mathrm{~b}$ University of Pretoria, South Africa. The Southern African Institute of Mining and Metallurgy, pages 1-11, 2015.

[14] Darren Turner, Arko Lucieer, and Christopher Watson. An automated technique for generating georectified mosaics from ultra-high resolution unmanned aerial vehicle (uav) imagery, based on structure from motion (sfm) point clouds. Remote Sensing, 4(5):1392-1410, 2012.

[15] MJ Westoby, J Brasington, NF Glasser, MJ Hambrey, and JM Reynolds. 'structure-from-motion' photogrammetry: A low-cost, effective tool for geoscience applications. Geomorphology, 179:300-314, 2012. 